\title{
ORIGINAL
}

\section{EVOLUCIÓN DEL USO DE MEDICAMENTOS ANSIOLÍTICOS E HIPNÓTICOS EN ESPAÑA DURANTE EL PERÍODO 2000-2011}

\author{
Maria Pilar Vicente Sánchez, Diego Macías Saint-Gerons, César de la Fuente Honrubia, Diana \\ González Bermejo, Dolores Montero Corominas y Ferrán Catalá-López.
}

División de Farmacoepidemiología y Farmacovigilancia. Agencia Española de Medicamentos y Productos Sanitarios (AEMPS). Madrid. España.

Las opiniones expresadas en este trabajo son responsabilidad de los autores, por lo que no reflejan necesariamente el punto de vista de los organismos en los que trabajan.

Conflictos de interés: Ninguno en relación a este trabajo.

\section{RESUMEN}

Fundamentos: Los ansiolíticos y los hipnóticos han sido durante años uno de los grupos farmacológicos más prescritos en la mayoría de países desarrollados. El objetivo principal del presente trabajo fue explorar el patrón de uso de ansiolíticos e hipnóticos en España durante el período 2000-2011 y comparar su incremento con el de cinco países europeos.

Método: Estudio ecológico descriptivo del consumo en España a parti de datos de medicamentos dispensados en oficina de farmacia y facturados a través de receta oficial con cargo al Sistema Nacional de Salud. El consumo anual y total se expresó en dosis diarias definidas por 1.000 habitantes y día (DDD/1.000 hab/día) en cada subgrupo terapéutico, principio activo y atendiendo a la semivida plasmática. También se establecieron comparaciones aproximativas con otros países.

Resultados: El uso de ansiolíticos e hipnóticos fue de 56,7 DDD/1.000 hab/día en el año 2000 y 82,9 DDD/1.000 hab/día en el año 2011 (incremento del $46,1 \%$ en el período). Lorazepam y alprazolam fueron los ansiolíticos más consumidos (20,5 y 15,6 DDD/1.000 hab/día en 2011, respectivamente), mientras que lormetazepam lo fue del grupo de los hipnóticos (18,3 $\mathrm{DDD} / 1.000 \mathrm{hab} . /$ día en 2011). En términos relativos, lormetazepam y zolpidem fueron los hipnóticos que más incrementaron su uso (103,3\% y $85,1 \%$, respectivamente) mientras que lorazepam e hidroxicina lo fueron entre los ansiolíticos $(75,1 \%$ y $72,8 \%$, respectivamente). En España (período 20032010 ), el incremento total en el consumo de ansiolíticos e hipnóticos fue de $+34,3 \%$, siendo del $+24,0 \%$ para Portugal, $+4,0 \%$ para Italia y $-6,1 \%$ para Francia.

Conclusiones: Durante el período estudiado, el consumo de ansiolíticos e hipnóticos se incrementó en España, siendo el incremento superior incluso al reportado en otros países europeos..

Palabras clave: Farmacoepidemiología. Consumo de medicamentos. Estudio de utilización. Ansiolíticos. Hipnóticos. España.

\section{Correspondencia}

Diego Macías Saint-Gerons

División de Farmacoepidemiología y Farmacovigilancia

Agencia Española de Medicamentos y Productos Sanitarios

Parque Empresarial "Las Mercedes"

Edificio $8,1^{\text {a }}$ planta

C/ Campezo, 1

28022 Madrid

Correo electrónico: dmacias.sg@gmail.com

\section{ABSTRACT}

\section{Trends of Use of Anxiolytics and} Hypnotics in Spain from 2000 to 2011

Background: For years, anxiolytics and hypnotics have been one of the most prescribed drug classes in most developed countries. The main aim of this study is to explore the pattern of use of anxiolytic and hypnotic drugs during the period 2000-2011, comparing their growth with that of five european countries.

Method: We performed an ecological and descriptive study of anxiolytics and hypnotics consumption in Spain. Consumption data were obtained from the databases of medications dispensed in community pharmacies and charged through official prescriptions to the totality of the Spanish National Health System. Annual and total-period consumptions were expressed in defined daily doses (DDD) per 1000 inhabitants per day (DDD/1000 person/day) by each treatment subgroup, active substance and attending the plasma half-life of the medication. Approximate comparisons were also made with some European countries.

Results: The use of anxiolytics and hypnotics drugs was $56.7 \mathrm{DDD} / 1000$ person/day in 2000 and $82.9 \mathrm{DDD} / 1000$ person/day in 2011 (a $+46.1 \%$ increase across the period). Lorazepam and alprazolam were the most used anxiolytics (20.5 and 15.6 DDD/1000 person/day in 2011, respectively), whereas lormetazepam was among the hypnotics (18.3 DDD/1000 person/day in 2011). In relative terms, hypnotics' lormetazepam and zolpidem increased their use by $103.3 \%$ and $85.1 \%$, respectively; while anxiolytics' lorazepam and hydroxyzine increased $75.1 \%$ and $72.8 \%$, respectively. In Spain (period 2003-2010), the total increase in the consumption of anxiolytics and hypnotics was $+34.3 \%$, with $24.0 \%$ for Portugal, $4.0 \%$ for Italy, but a reduction of $-6.1 \%$ for France.

Conclusions: A considerable increase in anxiolytics and hypnotics' consumption has occurred in Spain during the last decade, being the growth higher than that reported in other European countries.

Keyword: Pharmacoepidemiology. Drug consumption. Drug utilization study. Anxiolytics. Hypnotics. Spain. 


\section{INTRODUCCIÓN}

Durante años, los medicamentos ansiolíticos y los hipnóticos han sido uno de los grupos farmacológicos mas prescritos en la mayoría de países desarrollados ${ }^{1,2}$. De hecho, diversos estudios ${ }^{3-5}$ han mostrado que el consumo de estos medicamentos aumentó considerablemente y de manera sostenida en España durante los años 90. Análisis más recientes sugieren que España estaría situada por encima de la media europea en consumo de ansiolíticos ${ }^{6,7}$. En una encuesta reciente sobre abuso de drogas se estima que la prevalencia de uso de hipnóticos sedantes en España durante el año 2011 fue del $11,4 \%$ en población general ${ }^{8}$.

Aunque estos medicamentos tienen indicaciones terapéuticas diversas, como son el tratamiento de las contracturas musculares, las distonías neurovegetativas o el tratamiento de las convulsiones en epilepsia, su uso principal es el tratamiento a corto plazo del insomnio y de los trastornos de ansiedad. Además, el consumo a largo plazo de estos psicofármacos está asociado a una serie de riesgos para la salud de la población ${ }^{9}$.

El objetivo principal del presente trabajo es describir y explorar el patrón de uso de ansiolíticos e hipnóticos en España durante la primera década del siglo XXI, para cada subgrupo terapéutico y principio activo y comparar su incremento con el de Francia, Italia, Portugal, Noruega y Dinamarca.

\section{MATERIAL Y MÉTODO}

Estudio ecológico descriptivo del consumo de ansiolíticos e hipnóticos en España durante el período 2000-2011.

Se seleccionaron dos subgrupos terapéuticos de la clasificación Anatomical Therapeutic Chemical Classification (ATC) de la Organización Mundial de la Salud (OMS): grupo N05B (fármacos ansiolíticos) y grupo
N05C (fármacos hipnóticos y sedantes), que fueron clasificados según su semivida plasmática en las distintas categorías: acción corta ( $<10$ horas), acción intermedia (entre 10-24 horas) y acción larga ( $>24$ horas).

Las medidas de análisis fueron las Dosis Diarias Definidas (DDD) consumidas por cada 1.000 habitantes y día (DDD/1000 hab/día) en cada subgrupo terapéutico y para cada principio activo. La DDD es la dosis diaria de mantenimiento para un medicamento en su principal indicación en adultos y se estableció utilizando la metodología del Collaborating Centre for Drug Statistics and Methodology (www.whocc.no) de la OMS para cada principio activo. En los principios activos o asociaciones que no disponen de una DDD establecida por la OMS se consideró la DDD como la recomendada en la ficha técnica del medicamento.

Los datos de consumo fueron cedidos por la Dirección General de Cartera Básica de Servicios del Sistema Nacional de Salud y Farmacia (www.msssi.gob.es), en cuyas bases de datos se recogen las recetas de cada presentación dispensada con cargo al Sistema Nacional de Salud (SNS). Estas bases de datos se generan a partir de las recetas oficiales del médico prescriptor (talonarios de recetas) y de la facturación de cada receta presentada por las oficinas de farmacia y contienen la descripción del medicamento dispensado (marca comercial, presentación, dosis, número de unidades por envase y precio de venta al público). Sin embargo, no se incluye la utilización en el ámbito hospitalario, en atención médica privada ni el consumo de medicamentos no financiados o dispensados sin receta.

Los denominadores poblaciones para cada año y para el total del período estudiado se obtuvieron del censo de la población residente en España, información que es elaborada por el Instituto Nacional de Estadística (www.ine.es). 
De manera exploratoria, cuando ello fue posible, también se obtuvieron datos de consumo de 5 países europeos de nuestro entorno (como son Italia, Francia, Portugal, Noruega y Dinamarca), a partir de los informes disponibles en los portales web de las correspondientes agencias regulado$\operatorname{ras}^{10-14}$.

Análisis estadístico. Se realizaron descripciones de los valores absolutos y frecuencias (incluyendo variaciones porcentuales en el período de estudio).

\section{RESULTADOS}

El consumo de ansiolíticos e hipnóticos en conjunto fue de 56,73 DDD/1.000 hab/día en el año 2000 y de 82,9 DDD/1.000 hab/día en el año 2011, que significó un incremento del $46,1 \%$ durante el período. El grupo de hipnóticos y sedantes experimentó un incremento del $66,2 \%$, mientras que el de los ansiolíticos aumentó un $37,3 \%$. Se observó un incremento soste- nido del $2 \%$ al 3,5\% anual, con excepción de los años 2004 y 2006, en los que el incremento fue del 5,9\% y 5,3\% respectivamente, y del 7\% en el el año 2008. Atendiendo a la duración de la acción, los ansiolíticos e hipnóticos más consumidos en términos absolutos fueron los de acción intermedia, seguidos de los de acción larga. El mayor incremento de uso se registró también en los de acción intermedia pero seguidos de los de acción corta $(60,8 \%$ y $30,8 \%$, respectivamente) (figura 1 ).

Los hipnóticos y sedantes más consumidos durante el año 2011 fueron lormetazepam y zolpiden con 18,3 y $7,5 \mathrm{DDD} / 1.000$ hab/día respectivamente. Estos fármacos fueron los que experimentaron un mayor incremento de uso durante del período estudio (103,3\% y $85,1 \%$ respectivamente). Por el contrario, triazolam, flunitrazepam, quazepam, loprazolam y clometiazol fueron los que experimentaron el descenso más significativo (tabla 1). El ansiolítico más consumido durante el año 2011 fue

Figura 1

Evolución del consumo de ansiolíticos e hipnóticos en España (2000-2011) según su semivida plasmática

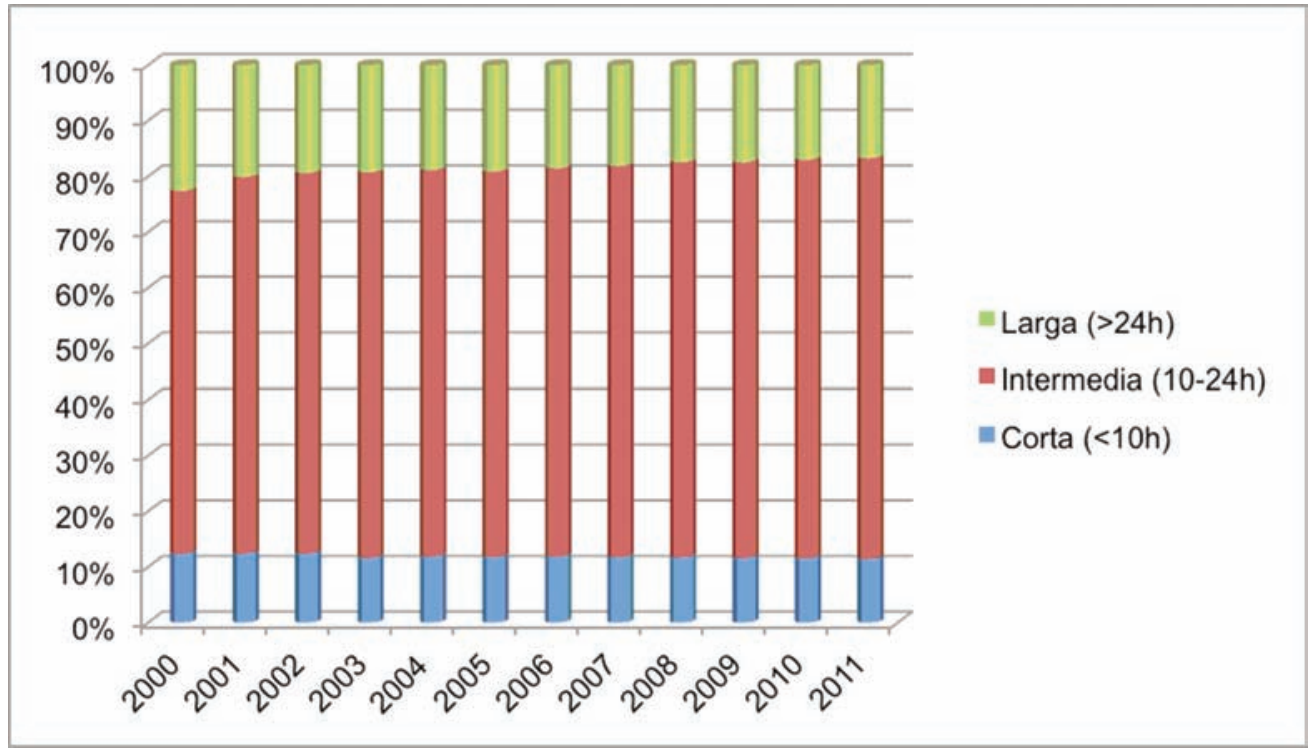


Tabla 1

Evolución durante el período 2000-2011 de la utilización de ansiolíticos e hipnóticos por principio activo expresado en DDD/1000 hab/ día

\begin{tabular}{|c|c|c|c|c|c|c|c|c|c|c|c|c|c|}
\hline Principio activo & 2000 & 2001 & 2002 & 2003 & 2004 & 2005 & 2006 & 2007 & 2008 & 2009 & 2010 & 2011 & $\begin{array}{c}\text { Incremento } \\
\%\end{array}$ \\
\hline \multicolumn{14}{|c|}{ Ansiolíticos (Grupo ATC N05B) } \\
\hline Alprazolam & 11,44 & 11,92 & 12,11 & 12,19 & 13,19 & 13,29 & 14,02 & 14,32 & 14,65 & 15,48 & 15,62 & 15,66 & 36,85 \\
\hline Bentazepam & 0,26 & 0,25 & 0,24 & 0,22 & 0,20 & 0,18 & 0,17 & 0,16 & 0,15 & 0,15 & 0,14 & 0,14 & $-48,03$ \\
\hline Bromazepam & 2,17 & 2,17 & 2,19 & 2,22 & 2,23 & 2,20 & 2,23 & 2,25 & 2,28 & 2,41 & 2,40 & 2,39 & 9,76 \\
\hline Clobazam & 0,37 & 0,36 & 0,34 & 0,33 & 0,34 & 0,33 & 0,33 & 0,33 & 0,33 & 0,34 & 0,34 & 0,35 & $-4,70$ \\
\hline Clorazepato dipotásico & 4,23 & 4,17 & 4,11 & 4,12 & 4,07 & 4,02 & 4,02 & 3,98 & 3,95 & 4,05 & 4,08 & 4,34 & 2,53 \\
\hline Clotiazepam & 0,18 & 0,18 & 0,17 & 0,16 & 0,15 & 0,15 & 0,14 & 0,14 & 0,14 & 0,14 & 0,14 & 0,13 & $-27,26$ \\
\hline Diazepam & 5,13 & 4,25 & 4,42 & 4,63 & 4,87 & 5,12 & 5,44 & 5,74 & 5,95 & 6,73 & 6,91 & 7,11 & 38,59 \\
\hline Halazepam & 0,52 & 0,46 & 0,46 & 0,47 & 0,47 & 0,47 & 0,48 & 0,48 & 0,49 & 0,53 & 0,53 & 0,53 & 2,37 \\
\hline Ketazolam & 1,32 & 1,40 & 1,50 & 1,52 & 1,51 & 1,47 & 1,44 & 1,41 & 1,39 & 1,41 & 1,37 & 1,34 & 1,71 \\
\hline Lorazepam & 11,72 & 12,69 & 13,63 & 14,29 & 15,28 & 15,87 & 16,85 & 17,64 & 18,34 & 19,71 & 20,31 & 20,52 & 75,13 \\
\hline Meprobamato & 0,01 & 0,01 & 0,01 & $<0,01$ & $<0,01$ & $<0,01$ & $<0,01$ & & & & & & - \\
\hline Oxacepam $^{\mathrm{a}}$ & 0,01 & $<0,01$ & $<0,01$ & $<0,01$ & $<0,01$ & $<0,01$ & $<0,01$ & & & & & & - \\
\hline Passiflora incarnata ${ }^{b}$ & & & & & & 0,03 & 0,03 & 0,03 & 0,03 & 0,03 & 0,03 & 0,04 & - \\
\hline Pinazepam & 0,07 & 0,07 & 0,05 & 0,04 & 0,03 & 0,03 & 0,03 & 0,03 & 0,03 & 0,03 & 0,03 & 0,03 & $-62,66$ \\
\hline Buspirona $^{c}$ & 0,02 & 0,02 & 0,02 & 0,02 & 0,02 & 0,02 & 0,02 & 0,02 & 0,01 & $<0,01$ & $<0,01$ & $<0,01$ & $-99,96$ \\
\hline Clorazepato dipotásico (combinaciones) ${ }^{\mathrm{a}} \mathrm{c}$ & 0,67 & 0,70 & 0,71 & 0,72 & 0,72 & 0,72 & 0,73 & 0,73 & 0,73 & 0,71 & 0,50 & $<0,01$ & $-99,54$ \\
\hline Clordiazepóxido $^{\mathrm{a}}$ & 0,06 & 0,01 & 0,01 & 0,01 & 0,01 & 0,01 & 0,01 & 0,01 & 0,01 & 0,01 & $<0,01$ & $<0,01$ & $-99,95$ \\
\hline Diazepam (combinaciones) & 0,76 & 0,69 & 0,64 & 0,64 & 0,89 & 1,32 & 1,27 & 1,13 & 0,84 & 0,84 & 0,79 & 0,75 & $-2,23$ \\
\hline Hidroxicina & 0,53 & 0,59 & 0,64 & 0,68 & 0,74 & 0,76 & 0,81 & 0,84 & 0,86 & 0,86 & 0,89 & 0,91 & 72,78 \\
\hline Total ansiolíticos & 39,48 & 39,94 & 41,22 & 42,25 & 44,71 & 45,98 & 48,03 & 49,25 & 50,17 & 53,43 & 54,09 & 54,22 & 37,34 \\
\hline \multicolumn{14}{|c|}{ Hipnóticos (Grupo ATC N05C) } \\
\hline Brotizolam & 0,23 & 0,21 & 0,20 & 0,18 & 0,17 & 0,16 & 0,16 & 0,15 & 0,15 & 0,15 & 0,15 & 0,14 & $-36,94$ \\
\hline Clometiazol & 0,20 & 0,20 & 0,18 & 0,00 & 0,21 & 0,22 & 0,23 & 0,23 & 0,24 & 0,25 & 0,26 & 0,15 & $-26,24$ \\
\hline Flunitrazepam $^{\mathrm{d}}$ & 0,80 & 0,67 & 0,60 & 0,55 & 0,51 & 0,48 & 0,46 & 0,44 & 0,43 & 0,43 & 0,42 & 0,40 & $-49,64$ \\
\hline Flurazepam & 0,73 & 0,73 & 0,72 & 0,68 & 0,70 & 0,70 & 0,69 & 0,69 & 0,69 & 0,72 & 0,70 & 0,69 & $-5,32$ \\
\hline Loprazolam & 1,14 & 1,01 & 0,95 & 0,89 & 0,85 & 0,78 & 0,74 & 0,71 & 0,69 & 0,71 & 0,69 & 0,66 & $-41,92$ \\
\hline Lormetazepam & 8,99 & 9,71 & 10,28 & 10,88 & 11,43 & 12,22 & 13,27 & 14,20 & 15,19 & 16,59 & 17,47 & 18,26 & 103,26 \\
\hline Midazolam & 0,18 & 0,18 & 0,17 & 0,17 & 0,17 & 0,17 & 0,18 & 0,19 & 0,19 & 0,20 & 0,21 & 0,21 & 18,62 \\
\hline Nitrazepam & $<0,01$ & $<0,01$ & $<0,01$ & $<0,01$ & $<0,01$ & $<0,01$ & & & & & & & - \\
\hline Quazepam & 0,09 & 0,08 & 0,07 & 0,06 & 0,06 & 0,05 & 0,05 & 0,05 & 0,05 & 0,05 & 0,05 & 0,05 & $-48,45$ \\
\hline Triazolam & 0,17 & 0,15 & 0,14 & 0,13 & 0,12 & 0,11 & 0,10 & 0,10 & 0,09 & 0,09 & 0,08 & 0,07 & $-59,09$ \\
\hline Zaleplón & & & 0,10 & 0,16 & 0,15 & 0,15 & 0,15 & 0,05 & $<0,01$ & $<0,01$ & $<0,01$ & $<0,01$ & - \\
\hline Zolpidem & 4,05 & 4,44 & 4,76 & 4,67 & 5,17 & 5,49 & 6,02 & 6,38 & 6,68 & 7,13 & 7,33 & 7,50 & 85,07 \\
\hline Zopiclona & 0,69 & 0,65 & 0,61 & 0,57 & 0,56 & 0,54 & 0,54 & 0,54 & 0,55 & 0,57 & 0,56 & 0,55 & $-20,32$ \\
\hline Total hipnóticos & 17,25 & 18,03 & 18,76 & 18,96 & 20,11 & 21,07 & 22,57 & 23,72 & 24,94 & 26,89 & 27,89 & 28,68 & 66,25 \\
\hline Total global & 56,73 & 57,96 & 59,99 & 61,21 & 64,83 & 67,05 & 70,60 & 72,97 & 75,11 & 80,33 & 81,98 & 82,90 & 46,13 \\
\hline
\end{tabular}

a) Excluido de la oferta del SNS. b) Excluido de la financiación en 2012. c) Autorización revocada en 2011 d) Suspendida comercialización en 2013. 
Figura 2

Consumo de ansiolíticos (N05B) e hipnóticos (N05C) en distintos países europeos durante el año 2010

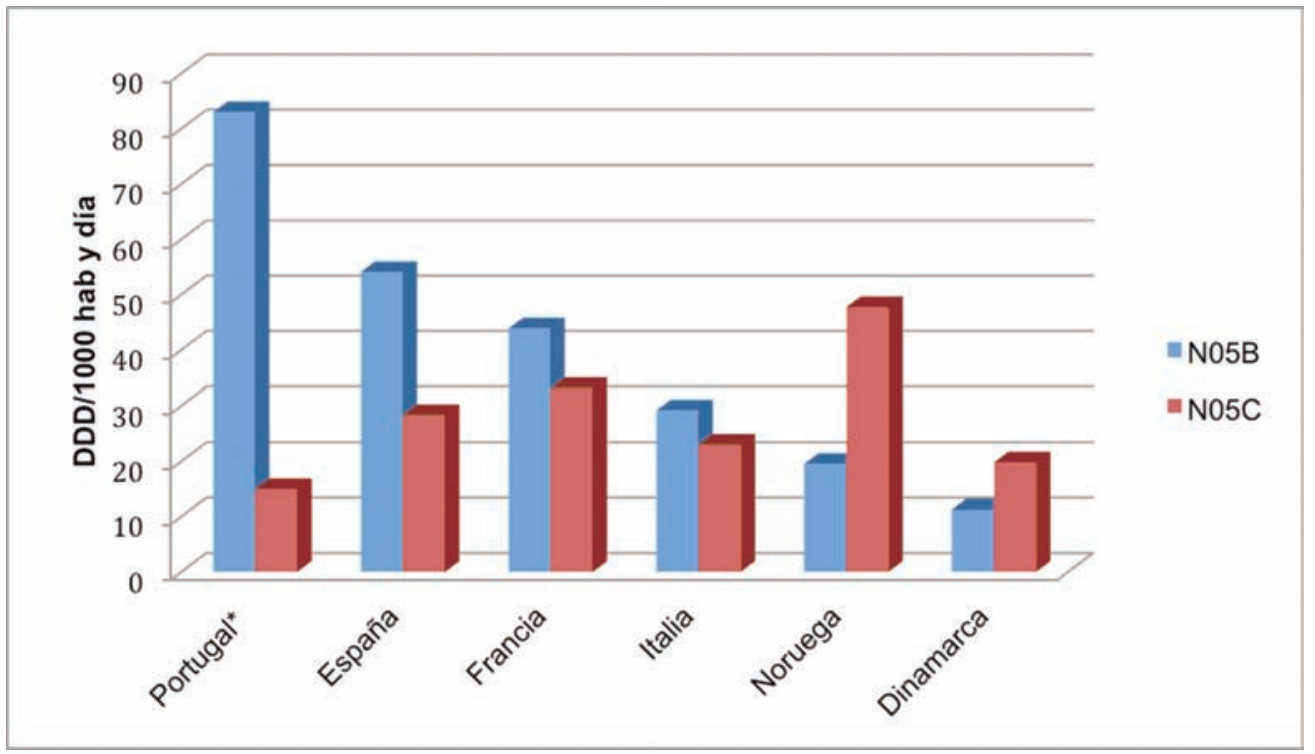

lorazepam (20,5 DDD/1000 hab/día) seguido de alprazolam (15,7 DDD/1000 hab/día). El incremento más importante se registró para lorazepam y hidroxizina ( $75,1 \%$ y $72,8 \%$, respectivamente), seguidos de alprazolam y diazepam $(38,6 \%$ y $36,9 \%$ respectivamente). Se observó un descenso en el uso de pinazepam, bentazepam, y clotiazepam (tabla 1).

\section{Tabla 2}

\section{Incremento de DDD/1000 hab y día de} ansiolíticos e hipnóticos durante el periodo 2003-2010 en diferentes países europeos mediterráneos

\begin{tabular}{|l|c|c|c|}
\hline PAÍS & $\begin{array}{c}\text { Incremento } \\
\text { N05B (\%) }\end{array}$ & $\begin{array}{l}\text { Incremento } \\
\text { N05C (\%) }\end{array}$ & $\begin{array}{c}\text { Incremento } \\
\text { total (\%) }\end{array}$ \\
\hline España & 28,01 & 47,16 & 33,94 \\
\hline Italia & $-3,31$ & 15,00 & 3,98 \\
\hline Francia & $-10,95$ & 1,22 & $-6,10$ \\
\hline Portugal* & 23,88 & 25 & 24,05 \\
\hline
\end{tabular}

*Periodo considerado 2003-2009
La comparación con el resto de países europeos permite destacar el mayor consumo de ansiolíticos con respecto a los hipnóticos en los países mediterráneos (España, Francia, Italia y Portugal) a diferencia de los países nórdicos (Noruega y Dinamarca) donde hay un consumo mayoritario de medicamentos hipnóticos y sedantes (figura 2). Por otra parte, en España durante el periodo 2003-2010, el incremento total en el consumo de ansiolíticos e hipnóticos fue de $+34,3 \%$, siendo superior al observado en Portugal $(+24,0 \%)$, Italia $(+4,0 \%)$ o Francia $(-6,1 \%)$ (tabla 2$)$.

\section{DISCUSIÓN}

Durante el período de estudio el consumo de ansiolíticos e hipnóticos, en especial bezodiazepinas y análogos, experimentó un incremento considerable y sostenido en España, pese a que no se han comercializado nuevos principios activos en ninguno de los grupos desde la autorización de zaleplón 
en 2001. Por el contrario en los últimos años han desparecido del mercado nitrazepam (2006), meprobamato (2007), combinaciones de clorazepato dipotásico (2011), buspirona (2011) y zaleplon, que se encuentra autorizado pero no se comercializa desde 2007.

Los principios activos más consumidos entre los ansiolíticos fueron lorazepam y alprazolam. Lorazepam está indicado en el tratamiento de la ansiedad asociada a la depresión y alprazolam es un ansiolítico con acción antidepresiva indicado también en el tratamiento de los trastornos de angustia. En España, la prevalencia de los trastornos de ansiedad y depresivos se sitúa entre el 4$8 \%{ }^{15,16}$ habiéndose observado un incremento de estos trastornos mentales como consecuencia de la actual crisis económica ${ }^{17}$. Teniendo en cuenta que los principales factores de riesgo para los trastornos del estado de ánimo se relacionan con aspectos socioeconómicos y laborales, es posible que se continúe registrando un aumento del consumo de psicofármacos en los próximos años. Es destacable que los ansiolíticos que experimentaron mayor aumento de utilización en los 3 últimos años son medicamentos con indicación en ansiedad asociada a depresión. Aunque en menor medida, en los años previos también se observó un aumento en la utilización de ansiolíticos con indicaciones en depresión. Todo ello podría reflejar el uso cada vez más frecuente de estos medicamentos en el tratamiento de los trastornos mixtos de ansiedad y depresión. En un estudio transversal realizado en España y otros 5 países europeos se observó que el diagnóstico de depresión mayor está asociado con la prescripción benzodiazepinas ${ }^{18}$. Es difícil valorar el incremento que ha experimentado hidroxicina, pues se trata de un antihistamínico indicado en el tratamiento de la ansiedad pero también en el tratamiento sintomático de prurito y urticaria, aunque su consumo en términos absolutos es bajo.

En términos absolutos los hipnóticos se consumen menos que el grupo de los ansiolíticos. Sin embargo, su consumo se ha incre- mentado en mayor medida que el de los ansiolíticos, especialmente a expensas de zolpidem y lormetazepam. Ambos están indicados en el tratamiento de corta duración del insomnio. Hay pocos estudios en España que hayan analizado la prevalencia del insomnio y no se han identificado datos publicados de incidencia en nuestro país. Las cifras de prevalencia del insomnio, en muestras de la población general, son variables en función de la definición o criterio diagnóstico utilizado. Según la clasificación del DSM-IV, la prevalencia de las personas con diagnóstico de insomnio en España se situaría alrededor del $6 \%$. Sin embargo cuando nos referimos a "quejas" sobre el sueño (o quejas de insomnio) las cifras pueden alcanzar el 30\% ${ }^{19}$. En un estudio realizado a través de encuestas en población general se observó una prevalencia del $20 \%$ de personas que presentaban al menos un síntoma de insomnio. La quinta parte de estas personas utilizaban tratamiento farmacológico para el insomnio ${ }^{20}$.

La tendencia hacia un consumo creciente de estos fármacos ya había sido observada en estudios similares realizados en los periodos $1987-1995^{4}$ y $1995-2002^{5}$. Si bien el uso de los hipnóticos, en especial zolpidem y lormetazepam, se incrementó en mayor medida que en el periodo precedente ${ }^{5}$, lorazepam, lormetazepam y alprazolam siguen siendo los ansiolíticos e hipnóticos más consumidos en España.

No obstante, la información presentada apunta que el consumo observado en este período ha situado a nuestro país entre los europeos con mayor consumo de estos medicamentos en 2011. En el sur de Europa se correspondería mayoritariamente a un uso del grupo "ansiolíticos" (N05B) mientras que en el norte correspondería en mayor medida del grupo "hipnóticos" (NO5C). En un estudio previo también se encontró un patrón de utilización similar con mayor prevalencia de uso de ansiolíticos en Italia y Francia en comparación con Alemania y Reino Unido ${ }^{21}$. 
Sin embargo, estos datos se deben tomar con cautela, porque la clasificación farmacológica (ATC) de hipnóticos y ansiolíticos podría no reflejar la indicación de uso real, como es el caso de lorazepam, que pertenece al grupo de ansiolíticos pero se utiliza también como hipnótico ${ }^{22}$.

Entre las limitaciones de este estudio cabe citarse que, por ser ecológico de carácter descriptivo, no permite valorar si la causa del consumo de medicamentos se debe al aumento del número de pacientes tratados (incluyendo formas leves), al aumento de la duración de los tratamientos o bien se debe a ambos factores.

La estimación del consumo a través del valor de la DDD que establece la OMS presenta otras limitaciones, ya que en ocasiones pueden existir diferencias entre el valor de la DDD establecido por la OMS y la dosis real utilizada en la práctica clínica. Sin embargo, esta unidad técnica de medida permite comparar los datos de consumo entre diferentes países. Otro aspecto a tener en cuenta es que el consumo real de estos medicamentos podría ser mayor que el reflejado en este estudio, ya que se excluyó la dispensación a cargo de receta privada y el denominador considerado fue el censo de la población española, una población más numerosa que la que recibe medicamentos con cargo al SNS.

En resumen, los resultados de este estudio, junto con datos de estudios anteriores, muestran como en los últimos 25 años no ha cesado el aumento en el consumo de estos medicamentos. La capacidad para producir dependencia de las benzodiazepinas hace, que en muchos casos, el consumo se prolongue durante meses, años e incluso décadas, en contraste con lo que aconsejan las guías de práctica clínica ${ }^{23}$. El elevado consumo en términos absolutos de alprazolam y lorazepam podría indicar una utilización cada vez mayor de estos medicamentos en trastornos del estado de ánimo. También es destacable el incremento de los hipnóticos lormetazepam y zolpiden. Aunque se necesitan estudios basados en datos individuales de pacientes para precisar los motivos que expliquen el aumento de su utilización, cabe recordar que el uso indiscriminado de estos medicamentos puede dar lugar a diversos problemas, como el deterioro de la memoria, el aumento del riesgo de accidentes de tráfico ${ }^{24} \mathrm{o}$ el incremento de las caídas y fracturas de cadera en ancianos ${ }^{25}$. La instauración de nuevos tratamientos con benzodiazepinas o análogos se debería restringir a los casos más graves en los que exista un trastorno intenso, que limite la actividad del paciente o en situaciones de estrés importante, no debiéndose prolongar su uso más allá de varias semanas.

\section{AGRADECIMIENTOS}

A la Dirección General de Cartera Básica de Servicios del Sistema Nacional de Salud y Farmacia, por facilitarnos la información relativa a los consumos de medicamentos que han sido utilizados en el presente trabajo.

\section{BIBLIOGRAFÍA}

1. Hollingworth SA, Siskind DJ. Anxiolytic, hypnotic and sedative medication use in Australia. Pharmacoepidemiol Drug Saf. 2010;19(3):280-8.

2. Lagnaoui R, Depont F, Fourrier A, Abouelfath A, Bégaud B, Verdoux H, et al. Patterns and correlates of benzodiazepine use in the French general population. Eur J Clin Pharmacol. 2004;60(7):523-9.

3. Martín Arias LH, Carvajal A, Martín De Diego I, De Abajo F. Before and alter triazolam: changes in the consumption of hypnotics in Spain. Br J Clin Pharmacol. 1995;40(3):289-90.

4. Rayón P, Montero D, Santamaría B, Madurga M, De Abajo FJ. Benzodiazepines consumption in Spain. Eur J Clin Pharmacol. 1997; 52: 321-3. 
5. García del Pozo J, De Abajo FJ, Carvajal A, Montero D, Madurga M, García del Pozo V. Utilización de ansiolíticos e hipnóticos en España (1995-2002). Rev Esp Salud Publica. 2004;78 (3): 379-387.

6. Simó Miñana J. Utilización de medicamentos en España y en Europa. Aten Primaria. 2012; 44(6):33547.

7. Khong TP, de Vries F, Goldenberg JS, Klungel OH, Robinson NJ, Ibáñez L, Petri H. Potential impact of benzodiazepine use on the rate of hip fractures in five large European countries and the United States Calcif Tissue Int. 2012;91(1):24-31.

8. Delegación del Gobierno para el Plan Nacional sobre Droga. Encuesta sobre alcohol y drogas en población general en España: EDADES 2011-2012. Madrid: Ministerio de Sanidad, Servicios Sociales e Igualdad;2013. Disponible en: http://www.pnsd.msc.es/Categoria2/observa/pdf/EDADES2011.pdf

9. Billioti de Gage S, Bégaud B, Bazin F, Verdoux H, Dartigues JF, Pérès K, Kurth T, Pariente A. Benzodiazepine use and risk of dementia: prospective population based study. BMJ. 2012;27;345

10. Gruppo di lavoro OsMed. L'uso dei farmaci in Italia. Rapporto nazionale anno 2011. Roma:Il Pensiero Scientifico Editore; 2012. Disponible en: http://www.agenziafarmaco.gov.it/it/content/luso-deifarmaci-italia-rapporto-osmed-2011

11. Cavalié P, Richard N (ed). État des lieux de la consommation des benzodiazépines en France - Rapport d'expertise-. Agence française de sécurité sanitaire des produits de santé (AFSSAPS); 2011. Disponible en: http://ansm.sante.fr/var/ansm site/storage/original/ap plication/3f1dc4756b5bc091879c9c254d95e05c.pdf

12. Furtado C, Ribeirinho M, Gaspar M. Análise da Evolução da Utilização de Psicofármacos em Portugal Continental entre 2000 e 2009. Autoridade Nacional do Medicamento e Produtos de Saúde, I. P. Dezembro 2010. Disponible en: http://www.infarmed.pt/portal/page/portal/INFARMED/MONITORIZACAO DO MERCADO/OBSERVATORIO/I NTRODUCAO_DE_FICHEIROS/Estudo_psicofarmacos.pdf

13. Sakshaug S, editor. Drug Consumption in Norway 2007-2011 (Legemiddelforbruket i Norge 2007-2011), Oslo, Norwegian Institute of Public Health; 2012 . Disponible en : http://www.fhi.no/eway/default.aspx?pid=240\&trg $=$ Content $6845 \&$ Main 6664 $=6839: 0: 25,7806: 1: 0$ : $0::: 0: 0 \&$ Content $6845=6689: 105330: 25,7806: 1: 68$ $41: 1::: 0: 0$
14. Sales of medicinal products within the different ATC groups in the primary healthcare sector. Statens Serum Institut; 2011 . Disponible en: http://www.ssi.dk/Sundhedsdataogit/Dataformidling/Laegemiddelstatistikker/Laegemiddelstatistik\%205\%20aar.aspx

15. Ministerio de Sanidad y Consumo. Estrategia en Salud Mental del Sistema Nacional de Salud 2006- 2007. Madrid: Ministerio de Sanidad y Consumo; 2007. Disponible en: www.msc.es/organizacion/sns/planCalidadSNS/pdf/excelencia/salud_mental/ESTRATEGIA_S ALUD MENTAL SNS PAG WEB.pdf

16. Haro JM, Palacin C, Vilagut G, Martinez M, Bernal M, Luque I, et al. Prevalencia de los trastornos mentales y factores asociados: resultados del estudio ESEMeD-Espana. Med Clin (Barc). 2006;126(12):445-51.

17. Gili M, Roca M, Basu S, McKee M, Stuckler D. The mental health risks of economic crisis in Spain: evidence from primary care centres, 2006 and 2010 Eur J Public Health. 2013; 23(1):103-8.

18. Demyttenaere K, Bonnewyn A, Bruffaerts R, De Girolamo G, Gasquet I, Kovess V, et al. Clinical factors influencing the prescription of antidepressants and benzodiazepines: results from the European study of the epidemiology of mental disorders (ESEMeD). J Affect Disord. 2008;110(1-2):84-93

19. Grupo de Trabajo de la Guía de Práctica Clínica para el Manejo de Pacientes con Insomnio en Atención Primaria. Guía de Práctica Clínica para el Manejo de Pacientes con Insomnio en Atención Primaria. Madrid: Agencia Laín Entralgo; 2009. Disponible en: http://www.guiasalud.es/GPC/GPC_465_Insomnio_L ain Entr compl.pdf

20. Ohayon MM, Sagales T. Prevalence of insomnia and sleep characteristics in the general population of Spain. Sleep Med. 2010;11(10):1010-8.

21. Ohayon MM, Lader MH. Use of psychotropic medication in the general population of France, Germany, Italy, and the United Kingdom. J Clin Psychiatry. 2002;63(9):817-25

22. Adán Valero, MA, Acín Gericó MT, Díaz Aguado J, Alejandre Lázaro G, Pérez Perales J. ¿Cómo utilizamos los hipnóticos en atención primaria? Semergen 2010;36(7):372-376.

23. Vicens Caldentey C, Fiol Gelabert F. Abordaje de la deshabituación de benzodiazepinas en atención primaria. Inf Ter Sist Nac Salud 2008; 32: 52-57. Disponible en: http://www.msc.es/biblioPublic/publicaciones/docs/vol32_2DeshabBenzodiaAtenPrimaria.pdf 
24. Dassanayake T, Michie P, Carter G, Jones A. Effects of benzodiazepines, antidepressants and opioids on driving: a systematic review and meta-analysis of epidemiological and experimental evidence. Drug Safety 2011; 34(2):125-156.

25. Macias D, Carvajal A. Los riesgos de los psicofármacos en el anciano. En: Manual básico de psiquiatría geriátrica. Valladolid, Universidad de Valladolid. Secretariado de Publicaciones e Intercambio Editorial; 2007 p.217227. 